Article Title:

\title{
OMICS TECHNOLOGY: FOODOMICS
}

\section{Authors:}

\section{Gerardo Alvarez-Rivera}

Laboratory of Foodomics

Institute of Food Science Research (CIAL-CSIC)

Nicolás Cabrera 9, Campus UAM Cantoblanco

28049 Madrid - Spain

email: gerardo.alvarez@csic.es

Phone: +34 910017819

\section{Said Toro Uribe}

Laboratory of Foodomics

Institute of Food Science Research (CIAL-CSIC)

Nicolás Cabrera 9, Campus UAM Cantoblanco

28049 Madrid - Spain

email: saidtorouribe@gmail.com

\section{Elena Ibañez}

Laboratory of Foodomics

Institute of Food Science Research (CIAL-CSIC)

Nicolás Cabrera 9, Campus UAM Cantoblanco

28049 Madrid - Spain

email: elena.ibanez@csic.es

Phone: +34-91-0017956

\section{Alejandro Cifuentes}

Laboratory of Foodomics

Institute of Food Science Research (CIAL-CSIC)

Nicolás Cabrera 9, Campus UAM Cantoblanco

28049 Madrid - Spain

email: a.cifuentes@csic.es

Phone: +34-91-0017955

\section{Monica Bueno}

Laboratory of Foodomics

Institute of Food Science Research (CIAL-CSIC)

Nicolás Cabrera 9, Campus UAM Cantoblanco

28049 Madrid - Spain

email: monica.bueno@csic.es

Phone: 34-91-0017900 


\section{Abstract:}

Foodomics is playing an essential role and is increasingly becoming a relevant multi-omic platform able to deal with traditional and new food challenges such as food safety, quality, traceability and health-diet interaction. In this context, innovative and high-throughput omic studies combined with bioinformatic tools are being developed for rapid identification of pathogens and multi-residue determination of contaminants, for understanding the interaction of bioactive compounds from diet, as well as for guaranteeing authenticity, traceability and quality of food products. This chapter aims to provide the reader with a general overview of the different areas of research covered by Foodomics, through the revision of state-of-the-art research works dealing with the application of the Foodomic approach to different aspects of Food Science including food safety, quality, traceability and health-diet interaction.

\section{Keywords:}

Foodomics; food science; proteomics; metabolomics; transcriptomics; health; food quality, food safety.

\section{Glossary}

Foodome: collection of all compounds present in any investigated food sample and/or in any biological system interacting with the investigated food at a given time.

Foodomics: A discipline that studies the food and nutrition domains through the application and integration of advanced omics technologies to improve consumer's well-being, health, and confidence. 


\section{Abbreviations:}

CA, carnosic acid;

CE, capillary electrophoresis;

CS, carnosol;

DLLME, dispersive liquid-liquid microextraction;

DNA, deoxyribonucleic acid;

ELISA, enzyme-linked immunosorbent assay;

$E R$, endoplasmic reticulum;

$F D$, fluorescence detection;

FT-ICR, fourier transform ion cyclotron resonance;

$\mathrm{GC}$, gas chromatography;

GCxGC, comprehensive two-dimensional gas chromatography;

$\mathrm{GM}$, genetically modified;

HPLC, high performance liquid chromatography;

HRMS, high resolution mass spectrometry;

IBD, inflammatory bowel disease;

$I C P$, inductively coupled plasma;

IMS, ion mobility spectrometry;

LC, liquid chromatography;

LIF, laser induced fluorescence detection;

MALDI, matrix-assisted laser desorption ionization;

MEKC, micellar electrokinetic chromatography;

$M R M$, multiple reaction monitoring;

$\mathrm{MS}$, mass spectrometry;

$\mathrm{MS} / \mathrm{MS}$, tandem mass spectrometry;

NMR, nuclear magnetic resonance;

PBMC, peripheral blood mononuclear cells;

$\mathrm{PCR}$, polymerase chain reaction;

$\mathrm{PDO}$, protected designation of origin;

PUFA, polyunsaturated fatty acid;

$\mathrm{QqQ}$, triple quadrupole;

qRT-PCR, quantitative real-time PCR

QuEChERS, quick easy cheap effective rugged and safe;

SDS-PAGE, sodium dodecyl sulfate-polyacrylamide gel electrophoresis;

SLE, solid-liquid extraction;

SPE, solid phase extraction;

TOF, time of flight;

UHPLC, ultra-high performance liquid chromatography;

WGS, whole genome sequencing;

RNA-seq, whole transcriptomic sequencing; 


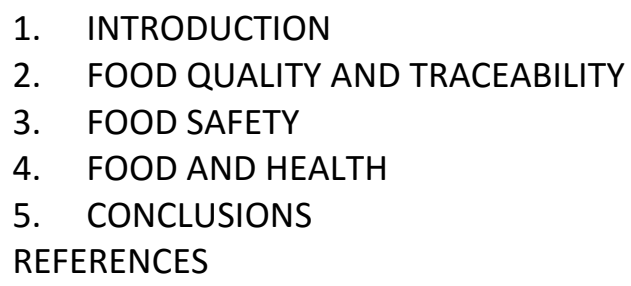

1. INTRODUCTION

2. FOOD QUALITY AND TRACEABILITY

3. FOOD SAFETY

4. FOOD AND HEALTH

5. CONCLUSIONS

REFERENCES

\section{Introduction}

Foodomics was defined for the first time in 2009 (Cifuentes, 2009) as "a discipline that studies the food and nutrition domains through the application and integration of advanced omics technologies to improve consumer's well-being, health, and confidence". Since its origin, the interest in Foodomics has greatly increased, showing a great adoption of the discipline as a useful and integrative framework to assess several crucial issues encountered in food science and nutrition, such as those related to food bioactivity, safety, quality and traceability (Fig. 1). Considering the complexity of Foodome (defined as "the collection of all compounds present in any investigated food sample and/or in any biological system interacting with the investigated food at a given time"), it is understandable the need of innovative, high-throughput, multi-omic platforms able to provide with the necessary data and information to offer real solutions and answers to the actual challenges in food science. As seen in Fig. 1, Foodomics involves not only gathering data coming from the different omics approaches (mainly, genomics, transcriptomics, proteomics, and metabolomics) but also the integration of all of them using advanced bioinformatics tools to be able to end up with the whole picture of the food-biological system interaction.

$<$ Figure 1 near here>

In this context, transcriptomics, proteomics and metabolomics represent powerful analytical platforms developed for the analysis of gene expression (transcripts), proteins and metabolites, 
respectively. Transcriptomics is commonly carried out using gene expression microarrays or massive sequencing of RNA (RNA-Seq), which makes possible the analysis of thousands of transcribed sequences quickly and efficiently (Morozova and Marra, 2008). Afterwards, gene validation, through quantitative RT-PCR (qRT-PCR), is normally employed (García-Cañas, Simó, León and Cifuentes, 2010). Two-dimensional gel electrophoresis followed by MALDI-TOF or TOF/TOF is the most widespread platform for Proteomic analysis (Griffiths and Grant, 2006), although other methods based on liquid chromatography (LC) (Valdes et al., 2017) or hybrid approaches (Dzieciatkowska, Hill and Hansen, 2014) have been also employed. On the other hand, a group of well-established analytical techniques, such as those based on NMR and MS, are the most frequently used in metabolic profiling and fingerprinting applications in metabolomics. These techniques are used either as standalone or, most commonly, combined with different separation techniques (LC-NMR, gas chromatography (GC)-MS, LC-MS, and capillary electrophoresis (CE)-MS). The combination of techniques produces complementary analytical information, thus allowing a wider metabolome coverage (Shulaev, 2006). By taking advantage of the newest methodological and instrumental developments related to omics techniques, Foodomics is continuously pushing the research in different hot topics in food science and nutrition such as: a) the comprehension of the biochemical, molecular and cellular mechanisms that underlies the beneficial or adverse effects of certain bioactive food components; b) the understanding of gene-based differences among individuals in response to a specific dietary pattern; c) the determination of the effect of bioactive food constituents on crucial molecular pathways; d) the knowledge of the identity of the genes involved in the previous stage to the onset of the disease, that can lead to the discovery of possible molecular biomarkers; e) the establishment of the global role and functions of gut microbiome; f) the investigation on unintended effects in genetically modified crops; g) the understanding of the stress adaptation responses of food-borne pathogens to ensure food hygiene, processing and preservation; h) the comprehensive assessment of food safety, quality and traceability ideally 
as a whole; i) the comprehension of the molecular basis of biological processes with agronomic interest and economic relevance, j) the roadmap towards a personalized nutrition, etc.

A good example of the interest of the scientific community in Foodomics is the quite large number of publications that have appeared in the last 4 years (more than 100 from different research groups in Europe, Asia and America). Some representative review papers on Foodomics are detailed in Table 1, covering aspects related to i) food quality and traceability, ii) food safety and ii) food bioactivity and health. Readers are encouraged to deepen in the different fields through these manuscripts and to get an overview of the most recent contributions to Foodomics in these areas through the reading of the present chapter.

<Table 1 near here>

\section{Food quality and traceability}

Food quality assessment is focused on multiple aspects, such as food appearance, origin, food composition, taste, flavor or food nutritional properties. These are important factor to be considered as they are most frequently used by consumers to judge food quality (Ryan, 2017). In this context, Foodomics represent a helpful approach to solve some of the new challenges that Food Science has to face in order to warranty food quality, and traceability.

MS-based metabolomics methodologies have demonstrated the potential to establish similarities and differences among different food products. For instance, metabolomic profiling using liquid chromatography coupled to tandem mass spectrometry (LC-MS) was successfully applied to monitor bioactive compounds as well as its bioactivity in several crop cultivars (e.g. cranberry, rice grain, tea extracts) (Brown, Murch and Shipley, 2012, Kim, Ha, Park et al., 2012, Oms-Oliu, Odriozola-Serrano and Martín-Belloso, 2013, Pongsuwan, Fukusaki, Bamba et al., 2007). Other untargeted studies based on GCXGC-TOF/MS and NMR have also been proposed for metabolomic profiling, focused on characterizing metabonomic changes across cranberry 
varieties from different origins (Humston, Zhang, Brabeck, McShea and Synovec, 2009, LópezRituerto, Savorani, Avenoza et al., 2012).

The huge potential of omic tools to solve new problems has also been implemented in seafood quality control. Proteomics analysis was shown to be a high throughput approach for identification of novel authenticity peptide biomarkers in a large number of samples with minimal time consumption (Jagadeesh, Kannegundla and Reddy, 2017). Studies combining twodimensional electrophoresis and MS protein identification have been used to establish fishery biomarkers in order to avoid food adulteration and prevent food fraud (Carrera, Cañas and Gallardo, 2013). The workflow for peptide biomarkers analysis of fishery products is depicted in Fig. 2

<Figure 2 near here>

Applications of proteomics together with NMR analysis, metabolomics and DNA-based methods can also be found in literature for food quality assessment (Korte and Brockmeyer, 2017). Some meat quality control procedures are based on off-flavor compounds identification, produced during meat lipid oxidation (Suman, Faustman, Stamer and Liebler, 2007), and the biochemical changes of pre- and post- mortem meat (Lametsch, Roepstorff and Bendixen, 2002). Moreover, genomic and transcriptomic tools have been implemented to investigate the nutritional potential of genus Lactobacillus plantarum A6 to improve the nutritional quality of basedfermented foods (Turpin, Weiman, Guyot et al., 2018). Other applications of transcriptomics with proteomics, metabolomics and statistical tools have been addressed to study food derived from genetically modified (GM) crops. For instance, Barros, Lezar, Anttonen et al. (2010), studied two GM maize varieties. Data revealed that environment and geographic conditions had a stronger overall effect on protein and gene expression than the genetic modification. In addition, the transcriptomic and metabolome data showed significant changes between the GM varieties and the non-GM maize variety. 
Food traceability is an essential aspect of foodomics, which provides precise information of food origin and food composition throughout all stages of the food supply chain; from farm production to consumer, through primary production, processing, distribution and retailing. This monitoring processes has often been defined as 'from farm to fork' (Montero, Herrero, Castropuyana and Raquel, 2017). The knowledge of the geographical origin, composition, processing method and distribution process of the end-food product are important aspects with strong influence on food quality. Therefore, new approaches using -omics technologies are necessary to provide precise and reliable traceability systems in order to warranty food quality and safety.

Advanced analytical techniques based on metabolomic fingerprinting have facilitated the traceability and authenticity of food products with Protected Designation of Origin (PDO). NMR and multivariate data analysis has been applied to different food products such as Mozzarella di Bufala Campana and Italian garlic to certify their PDO and authenticity (Mazzei and Piccolo, 2012, Ritota, Casciani, Han et al., 2012). The use of chemical markers to trace the production chain of food products was applied to assess wine production traceability. Aceto, Robotti, Oddone et al. (2013) performed ICP-MS analysis to Moscato grape samples and suggested that traceability seems possible since the lanthanides distribution is maintained from soil to grape and must. Other studies implemented $\mathrm{X}$-ray, carbon ${ }^{13} \mathrm{C} /{ }^{12} \mathrm{C}$ and nitrogen ${ }^{15} \mathrm{~N} /{ }^{14} \mathrm{~N}$ isotopes ratios to identify geographical markers on unifloral honey (Kropf, Korošec, Bertoncelj et al., 2010). Similar chemometric methods have been employed to determine geographic origin and for classification of green teas (Chen, Yu, Xu, Chen and Shi, 2009, Ren, Wang, Ning et al., 2013), vinegar (Ríos-Reina, García-González, Callejón and Amigo, 2018), potatoes (Latorre, García, Martín and Crecente, 2013), coffee (Barbosa, Batista, Varrique et al., 2014), mushrooms (Yao, Li, Liu, Li and Wang, 2018), and cocoa beans (Bertoldi, Barbero, Camin, Caligiani and Larcher, 2016, Marseglia, Acquotti, Consonni et al., 2016). 
Other methodologies include DNA-based methods for traceability applications. For instance, amplified nuclear DNA fragments (>150 bp) were used to analyze the quality and quantity of steak genomic DNA samples cooked at $100{ }^{\circ} \mathrm{C}$ (Aslan, Hamill, Sweeney, Reardon and Mullen, 2009). Implementation of DNA barcoding has also been proved for seafood traceability. The increasing demand of seafood and the inclusion of new fish species have been addressed to

implement control systems. Thereby, the Fish Barcode of Life Initiative (http://www.fishbol.org/), with more than 70,000 barcode sequences from 8,300 species, contains links to voucher specimens, information on species distribution and taxonomic information (Galimberti, De Mattia, Losa et al., 2013). This tool has also been useful for the identification of fish products sold in parts (Di Pinto, Marchetti, Mottola et al., 2015).

\section{Food safety}

Food safety is one of the main challenges for today's world due to the growing consumers concern about what is in their food and the safety of the food they eat. Food safety assessment involves the examination of food for the presence of microbial hazards induced by toxins, bacteria, viruses and parasites, allergens, as well as chemical contaminants such as agrochemicals, environmental contaminants and veterinary drugs, among others (Ibanez, Simo, Garcia-Canas, Acunha and Cifuentes, 2015).

Nutritional trends and globalization of food production and distribution are substantially forcing the establishment of restrictive maximum residue levels promoted by governments and international organizations. Instrumentation and Foodomics approaches represent a powerful tool for food safety assessment to face the challenge of detecting potentially dangerous compounds and microorganisms present in food at levels lower than the official requirements, as evidenced by the growing number of published reports in this regard. 
Most applications and methods devoted to food assessment take advantage of the capabilities of coupled techniques. MS or IMS detection can be easily coupled to separation techniques such as HPLC, GC or CE for the analysis of food related molecules including biogenic amines, contaminants and veterinary drugs, DNAs, food additives, pesticides and toxins (Alvarez, Montero, Llorens, Castro-Puyana and Cifuentes, 2018, Castro-Puyana, Mendiola, Ibáñez and Herrero, 2013, Karpas, 2013). Since food samples are complex matrices, different sample preparation techniques to isolate food toxins or contaminants are normally applied. Immunocapture, precipitation, filtration, SPE, SLD, SDS-PAGE, QuEChERS extraction or DLLME are the most common methodologies for sample preparation.

\subsection{Pathogens and toxins}

According to the World Health Organization (WHO), Salmonella, Campylobacter, and Enterohaemorrhagic Escherichia coli (E. coli) are the most prevalent foodborne pathogens that affect millions of people annually (World Health Organization, 2015), while Listeria monocytogenes contamination is of primary concern in processed products (Tompkin, 2002). The identification, confirmation, and quantification of bacteria and bacterial toxins in food are important analytical problems. Fortunately, to identify the harmful microorganisms in food with a quick and reliable way, omics methods offer ways forward. As an example, WGS, RNA-seq, CE and combination of molecular techniques with other analytical approaches, such as multiplexPCR-CE-LID or MALDI-TOF-MS, have been used for the detection, identification and characterization of pathogens (Resetar, Pavelic and Josic, 2015). For instance, use of RNA-seq technology has demonstrated its high utility in food safety studies such as the specific molecular mechanisms underlying the survival and persistence of Salmonella enterica in low water activity foods (Deng, Li and Zhang, 2012). Fig 3 shows a comparative transcriptomic map of E. Enteritidis cells inoculated in broth and in peanut oil. 
<Figure 3 near here>.

Poisonings caused by toxic components, such as mycotoxins that come in the soil, water and air, are sometimes difficult to link to a particular food. Mycotoxins are secondary metabolites produced by certain molds that exert toxic effects on humans. They can grow on a variety of different crops and foodstuffs such as grains and seeds, often under warm and humid conditions. Examples of mycotoxins of greatest public health and agro-economic significance include aflatoxins, ochratoxins, trichothecenes, zearalenone and fumonisins. The commonly used techniques for the quantitation of mycotoxins in targeted metabolomics are LC-QqQ-MS and LC-TOF-MS, while UHPLC-HRMS or FT-ICR-MS are preferred for the identification in nontargeted metabolomics (Rychlik, Kanawati and Schmitt-Kopplin, 2017).

Saxitoxin is one of the dangerous paralytic shellfish toxins whose determination is crucial in order to ensure the safety of seafood consumption. This toxin can be determined by HPLC-FD, MEKC-LIF or CE (Alvarez, Montero, Llorens, Castro-Puyana and Cifuentes, 2018).

\subsection{Allergens}

A food allergy reaction is an adverse health effect arising from an overreaction of the immune system to certain food components. According to the American College of Allergy, Asthma \& Immunology (ACAAI), eight types of food account for about the $90 \%$ of all reactions (eggs, milk, peanuts, tree nuts, fish, shellfish, wheat and soy) (American College of Allergy Asthma \& Immunology, 2014).

Food allergens are proteins or glycoproteins basically resistant to digestive enzymes in the gastrointestinal tract and heat treatment. Therefore, proteomics integrated with clinical, immunological, genomics and bioinformatics approaches provides an invaluable contribution to disclose many key aspects concerning food allergies (Picariello, Mamone, Addeo, Nitride and 
Ferranti, 2013). Immunological methods such as ELISA, DNA-based methods, MS-based proteomics or antibody and DNA-based biosensors can be used for the determinations of allergens in food (Andjelkovic, Gavrovic-Jankulovic, Martinovic and Josic, 2017).

\subsection{Chemical contaminants}

Residues of pesticides and veterinary drugs are the most investigated contaminants in food from plants and animal origin, respectively (Gilbert-López, Valdés, Acunha et al., 2017). The most frequent analytical approach to determine contaminants in foods relies on the use of tandem MS detection.

LC-MS/MS with a triple quadrupole operating in MRM mode or GC-MS/MS methods have been widely employed to simultaneously monitor more than 100 compounds in less than 40 min in olives (Gilbert-Lopez, Garcia-Reyes, Lozano, Fernandez-Alba and Molina-Diaz, 2010), egg, honey, milk (Robert, Gillard, Brasseur et al., 2013), chicken, fish, pork, rabbit (Zhang, Wang, Li and Wang, 2017), ginseng, spinach (Hayward, Wong, Zhang et al., 2011), cowpea (Yongtao, Le, Nan et al., 2016), cardamom (Shabeer, Girame, Utture et al., 2018) and tea (Hou, Lei, Guo and Qiu, 2016).

\section{Food and health}

In recent years, the prevention of human diseases by means of the proper diet control and the intake of functional food or nutraceutical products is becoming an emerging trend in medicine, food and bioscience fields. In this context, Foodomics is a powerful tool to understand the interaction of bioactive compounds from diet at molecular and cellular level, as well as to provide better scientific evidences of the benefits that food bioactive compounds can have on human's heath. Combining the data obtained from the three expression levels (gen, protein and 
metabolite), Foodomics can play a major role on nutrition and health research by detecting small changes induced by food ingredients at different expression levels (Ibáñez and Cifuentes, 2014).

The integration of transcriptomic, proteomic and metabolomic data is a challenging task, which requires the application of multiple analytical platforms along with advanced bioinformatics and statistical tools to tackle this holistic approach. Therefore, the number of studies reported in literature applying an integrated strategy to the foodomic study of bioactive compounds is still scarce, although some authors have already addressed the integration of several omics platforms for the study of the effects of dietary components in human health.

In recent years, great efforts were focused on the foodomic evaluation of the dietary bioactives effect on colorectal cancer prevention. In this regard, the potential of the Foodomics strategy was demonstrated for the first time by Ibanez, Valdes, Garcia-Canas et al. (2012), through a case study about the chemopreventive effect of dietary polyphenols against human colon cancer cells (see the proposed global foodomics strategy in Fig 4A). Differentially expressed genes, proteins, and metabolites in control and HT-29 colon cancer cells treated with rosemary extract were identified. The integration of the outcomes from the three omic platforms, based on the networking and mapping capabilities of Ingenuity Pathway Analysis (IPA) software, revealed the cellular mechanisms involved in the chemopreventive effect of the rosemary extract (apoptosis and cell cycle arrest; Fig. 4B). Further research on the bioactivity of rosemary extract against colon cancer HT-29 cells revealed the additive antiproliferative effect of carnosic acid (CA) and carnosol (CS); two major diterpens in rosemary extracts (Valdes, Garcia-Canas, Simo et al., 2014). At the transcriptomic level, CA was shown to induce transcriptional activation of genes that encode detoxifying enzymes in the colon cancer cell line. Colon cancer cells challenged with CA exhibited alteration in the expression levels of genes linked to transport and terpenoids biosynthesis functions. The results from metabolomic analysis revealed high levels of reduced 
glutathione in CA treated cells, evidencing the chemopreventive response of cells to CA treatment. A subsequent proteomic study identified the differences between the mechanisms of action of CA and CS in colon cancer cells. In light of the results obtained from the MS-based quantitative proteomics using stable isotope dimethyl labeling, CA treatment was shown to induce the expression of proteins involved in the unfolded protein response as a result of the ER stress, whereas CS directly inhibits chymotrypsin-like activity of the 20 S proteasome (Valdes, Garcia-Canas, Artemenko et al., 2017).

\section{<Figure 4 near here>.}

Polyphenol enriched supercritical rosemary extract was also shown to induce cholesterol accumulation after $24 \mathrm{~h}$ treatment of colon cancer cells, as reported by Valdés, Sullini, Ibáñez, Cifuentes and García-Cañas (2015) in a targeted metabolomics analysis. Complementary results from the transcriptomic study suggest up-regulation of VLDLR as the main gene responsible for the observed cholesterol accumulation, which contributes to an important strategy to block cancer cell growth. Additionally, transcriptomics revealed induction of unfolded protein response and ER stress on treated cells. This behavior of polyphenol-enriched rosemary extract was further confirmed though a comprehensive proteomic study, which showed changes in proteins implicated in the activation of Nrf2 transcription factor and the unfolded protein response, which is related to the activation of autophagy to alleviate cellular stress (Valdés, Artemenko, Bergquist, García-Cañas and Cifuentes, 2016). The benefits of dietary polyphenols form rosemary were also studied on leukemia cells. Valdes, Simo, Ibanez et al. (2012) reported the inhibition of Myc transcription factor on treated cells, which might be explained by the antiproliferative effect of rosemary polyphenols. Additional evidences to transcriptomic results were provided by the metabolomics outcomes, showing increased levels of GSH and decreased levels of hypoxanthine. Altogether, the results demonstrated the differential chemopreventive effect of rosemary polyphenols in leukemia cells with different phenotypes. 
Another integrated study, focused at transcriptomic and proteomic level, evaluated the effect of dietary selenium in colorectal cancer prevention (Meplan, Johnson, Polley et al., 2016). Analyzed RNA and proteins, obtained from rectal biopsy tissues, showed that the most significantly affected canonical pathways were involved in inflammatory and immune signaling, suggesting an overall down-regulation of immune response in individuals with suboptimal Se status. Results of proteomic observations were consistent with the RNA expression analysis, as evidenced by the interconnection of several networks under an integrated IPA mapping. Nuclear factor NFkB, factors IL1, TNF, and Akt, along with proto-oncogenes c-Myc, c-Fos and b-catenin were identified as central nodes of interconnected proteomic and microarray networks.

The use of parsley as bioactive source for inflammatory bowel disease (IBD) was studied in a nutraceutical intervention survey by Jia, Aw, Hanate et al. (2014). Following a multi-omics integrated strategy, this study was carried out using a C57BL/6J colitis induced-mice model. Colon transcriptome of treated mice revealed a decrease in the expression of inflammatory cytokines and fibrotic markers, resulting in reduced inflammation and improved colon shortening. Down-regulated cancer markers were observed in the hepatic transcriptome and proteome, as well as increases in the expression of fatty acid synthesis related to body weight loss, whereas complementary metabolomic data showed upregulation of molecules involved in the citric acid and urea cycles suggesting recovery in both glycolysis and oxidative stress, suggesting improvement in glycolysis and oxidative stress. In a previous study the immunemodulating effects of kiwifruit extracts (KFE) against IBD was also tested in a mouse model. The results of the gene and protein expression level profiling showed a subtle effect of kiwi extracts on immune signaling pathways and metabolic processes in the colon tissue, which suggest that KFE may have beneficial activity within the adaptive immune system (Edmunds, Roy, Davy et al., 2012). 
The effects of an anti-inflamatory diet on overweight and obesity were also studied under a foodomic approach (Bakker, van Erk, Pellis et al., 2010). The integrated analysis of a multi-omic study indicated modulated inflammation of adipose tissue, improved endothelial function, affected oxidative stress, and increased liver fatty acid oxidation. The anti-inflammatory dietary mix of this nutritional intervention study consisted of fish oil, green tea extract, resveratrol, vitamin $E$, vitamin $C$, and tomato. Plasma and urine metabolomics, along with the lipid and free fatty acid profiling analysis were combined with proteomics and transcriptomic data from peripheral blood mononuclear cells (PBMC) and adipose tissue samples. In other study, using supplementation of $n-3$ polyunsaturated fatty acids with or without fish gelatin, transcriptomics of PBMC and metabolomics analysis of plasma demonstrated the anti-inflammatory effect of $n$ 3 PUFA supplementation in PBMCs of 16 obese insulin-resistant subjects (Rudkowska, Ponton, Jacques et al., 2011).

\section{Conclusions}

In recent years, Foodomics has been successfully adopted by several authors to evaluate food safety, quality, traceability and the effect of bioactive food compounds in the control and prevention of human diseases. The use of data obtained from transcriptomic, proteomic and metabolomics analysis was demonstrated to be a powerful tool to corroborate food safety, quality and traceability as well as for the study of small changes induced by food ingredients at the different expression levels. These emerging multi-omic studies are contributing in a crucial manner to understand the different mechanisms involved in the bioactivity of food ingredients and nutraceuticals on health. 
Nevertheless, some limitations are slowing down the research activity in this field, such as lack of computational tools and biostatistical methods, the existing limited knowledge of all the cellular processes at the molecular level, and metabolomic databases need to be completed and improved to conveniently identify the wide range unknown metabolites detected in metabolomic studies. Overcoming these challenges will allow scientist to gain a comprehensive foodomic insight of the relation between food and health, while reinforcing the control of food safety, quality and traceability.

\section{REFERENCES:}

Aceto, M., Robotti, E., Oddone, M., Baldizzone, M., Bonifacino, G., Bezzo, G., Di Stefano, R., Gosetti, F., Mazzucco, E., Manfredi, M. and Marengo, E. (2013). A traceability study on the Moscato wine chain. Food Chemistry 138, 1914-1922.

Alvarez, G., Montero, L., Llorens, L., Castro-Puyana, M. and Cifuentes, A. (2018). Recent advances in the application of capillary electromigration methods for food analysis and Foodomics. Electrophoresis 39, 136-159.

American College of Allergy Asthma \& Immunology. (2014). Food allergy [Online]. Available: https://acaai.org/allergies/types/food-allergy [Accessed 19 April 2018].

Andjelkovic, U., Gavrovic-Jankulovic, M., Martinovic, T. and Josic, D. (2017). Omics methods as a tool for investigation of food allergies. Trac-Trends in Analytical Chemistry 96, 107-115.

Andjelković, U., Šrajer Gajdošik, M., Gašo-Sokač, D., Martinović, T. and Josić, D. (2017). Foodomics and Food Safety: Where We Are. Food Technology and Biotechnology 55, 290-307.

Aslan, Ö., Hamill, R. M., Sweeney, T., Reardon, W. and Mullen, A. M. (2009). Integrity of nuclear genomic deoxyribonucleic acid in cooked meat: Implications for food traceability. Journal of Animal Science 87, 57-61.

Bakker, G. C. M., Van Erk, M. J., Pellis, L., Wopereis, S., Rubingh, C. M., Cnubben, N. H. P., Kooistra, T., Van Ommen, B. and Hendriks, H. F. J. (2010). An antiinflammatory dietary mix modulates inflammation and oxidative and metabolic stress in overweight men: a nutrigenomics approach. The American Journal of Clinical Nutrition 91, 1044-1059.

Barbosa, R. M., Batista, B. L., Varrique, R. M., Coelho, V. A., Campiglia, A. D. and Barbosa, F. (2014). The use of advanced chemometric techniques and trace element levels for controlling the authenticity of organic coffee. Food Research International 61, 246-251.

Barros, E., Lezar, S., Anttonen, M. J., Van Dijk, J. P., Röhlig, R. M., Kok, E. J. and Engel, K. H. (2010). Comparison of two GM maize varieties with a near-isogenic non-GM variety using transcriptomics, proteomics and metabolomics. Plant biotechnology journal 8, 436-451.

Bayram, M. and Gokirmakli, C. (2018). Horizon Scanning: How Will Metabolomics Applications Transform Food Science, Bioengineering, and Medical Innovation in the Current Era of Foodomics? Omics 22, 177-183.

Bertoldi, D., Barbero, A., Camin, F., Caligiani, A. and Larcher, R. (2016). Multielemental fingerprinting and geographic traceability of Theobroma cacao beans and cocoa products. Food Control 65, 46-53. 
Braconi, D., Bernardini, G., Millucci, L. and Santucci, A. (2018). Foodomics for human health: current status and perspectives. Expert Rev Proteomics 15, 153-164.

Brown, P. N., Murch, S. J. and Shipley, P. (2012). Phytochemical diversity of cranberry (Vaccinium macrocarpon Aiton) cultivars by anthocyanin determination and metabolomic profiling with chemometric analysis. Journal of Agricultural and Food Chemistry 60, 261-271.

Canela, N., Rodriguez, M. A., Baiges, I., Nadal, P. and Arola, L. (2016). Foodomics imaging by mass spectrometry and magnetic resonance. Electrophoresis 37, 1748-67.

Carrera, M., Cañas, B. and Gallardo, J. M. (2013). Proteomics for the assessment of quality and safety of fishery products. Food research international 54, 972-979.

Castro-Puyana, M., Mendiola, J. A., Ibáñez, E. and Herrero, M. (2013). MS-based metabolomics approaches for Food Safety, Quality and Traceability. In: Cifuentes, A. (ed.) Foodomics: Advanced Mass Spectrometry in Modern Food Science and Nutrition. pp 453-470. New Jersey: John Wiley \& Sons.

Chen, Y., Yu, M., Xu, J., Chen, X. and Shi, J. (2009). Differentiation of eight tea (Camellia sinensis) cultivars in China by elemental fingerprint of their leaves. Journal of the Science of Food and Agriculture 89, 2350-2355.

Cifuentes, A. (2009). Food analysis and Foodomics. Journal of Chromatography A 1216, 7109.

Cifuentes, A. (2015). Foodomics technologies: Foodomics: exploring safety, quality and bioactivity of foods in the 21st century. Current Opinion in Food Science 4, 136-138.

Deng, X., Li, Z. and Zhang, W. (2012). Transcriptome sequencing of Salmonella enterica serovar Enteritidis under desiccation and starvation stress in peanut oil. Food Microbiology 30, 311-315.

Di Pinto, A., Marchetti, P., Mottola, A., Bozzo, G., Bonerba, E., Ceci, E., Bottaro, M. and Tantillo, G. (2015). Species identification in fish fillet products using DNA barcoding. Fisheries Research 170, 9-13.

Dzieciatkowska, M., Hill, R. and Hansen, K. C. (2014). GeLC-MS/MS analysis of complex protein mixtures. Methods Mol Biol 1156, 53-66.

Edmunds, S. J., Roy, N. C., Davy, M., Cooney, J. M., Barnett, M. P., Zhu, S., Park, Z., Love, D. R. and Laing, W. A. (2012). Effects of kiwifruit extracts on colonic gene and protein expression levels in IL-10 gene-deficient mice. Br J Nutr 108, 113-29.

Galimberti, A., De Mattia, F., Losa, A., Bruni, I., Federici, S., Casiraghi, M., Martellos, S. and Labra, M. (2013). DNA barcoding as a new tool for food traceability. Food Research International 50, 55-63.

Gallo, M. and Ferranti, P. (2016). The evolution of analytical chemistry methods in foodomics. J Chromatogr A 1428, 3-15.

García-Cañas, V., Simó, C., León, C. and Cifuentes, A. (2010). Advances in Nutrigenomics research: Novel and future analytical approaches to investigate the biological activity of natural compounds and food functions. Journal of Pharmaceutical and Biomedical Analysis 51, 290-304.

Gilbert-Lopez, B., Garcia-Reyes, J. F., Lozano, A., Fernandez-Alba, A. R. and Molina-Diaz, A. (2010). Large-scale pesticide testing in olives by liquid chromatography-electrospray tandem mass spectrometry using two sample preparation methods based on matrix solid-phase dispersion and QuEChERS. Journal of Chromatography A 1217, 6022-6035.

Gilbert-López, B., Mendiola, J. A. and Ibáñez, E. (2017). Green foodomics. Towards a cleaner scientific discipline. TrAC Trends in Analytical Chemistry 96, 31-41.

Gilbert-López, B., Valdés, A., Acunha, T., García-Cañas, V., Simó, C. and Cifuentes, A. (2017). Chapter 10 - Foodomics: LC and LC-MS-based omics strategies in food science and nutrition A2 - Fanali, Salvatore. In: Haddad, P. R., Poole, C. F. \& Riekkola, M.-L. (eds.) Liquid Chromatography (Second Edition). pp 267-299. Elsevier.

Griffiths, H. R. and Grant, M. M. (2006). The use of proteomic techniques to explore the holistic effects of nutrients in vivo. Nutr Res Rev 19, 284-93. 
Hayward, D. G., Wong, J. W., Zhang, K., Chang, J., Shi, F., Banerjee, K. and Yang, P. (2011). Multiresidue Pesticide Analysis in Ginseng and Spinach by Nontargeted and Targeted Screening Procedures. Journal of Aoac International 94, 1741-1751.

Hou, X., Lei, S., Guo, L. and Qiu, S. (2016). Qptimization of a multi-residue method for 101 pesticides in green tea leaves using. gas chromatography-tandem mass spectrometry. Revista Brasileira De Farmacognosia-Brazilian Journal of Pharmacognosy 26, 401-407.

Humston, E. M., Zhang, Y., Brabeck, G. F., Mcshea, A. and Synovec, R. E. (2009). Development of a GCXGC-TOFMS method using SPME to determine volatile compounds in cacao beans. Journal of Separation Science 32, 2289-2295.

Ibanez, C., Simo, C., Garcia-Canas, V., Acunha, T. and Cifuentes, A. (2015). The role of direct highresolution mass spectrometry in foodomics. Analytical and Bioanalytical Chemistry 407, 6275-6287.

Ibanez, C., Valdes, A., Garcia-Canas, V., Simo, C., Celebier, M., Rocamora-Reverte, L., GomezMartinez, A., Herrero, M., Castro-Puyana, M., Segura-Carretero, A., Ibanez, E., Ferragut, J. A. and Cifuentes, A. (2012). Global Foodomics strategy to investigate the health benefits of dietary constituents. J Chromatogr A 1248, 139-53.

Ibáñez, E. and Cifuentes, A. (2014). Chapter 15 - Foodomics: Food Science and Nutrition in the Postgenomic Era. In: García-Cañas, V., Cifuentes, A. \& Simó, C. (eds.) Comprehensive Analytical Chemistry. pp 395-440. Elsevier.

Jagadeesh, D. S., Kannegundla, U. and Reddy, R. K. (2017). Application of proteomic tools in food quality and safety. Advances in Animal and Veterinary Sciences 5, 213-225.

Jia, H., Aw, W., Hanate, M., Takahashi, S., Saito, K., Tanaka, H., Tomita, M. and Kato, H. (2014). Multi-faceted integrated omics analysis revealed parsley (Petroselinum crispum) as a novel dietary intervention in dextran sodium sulphate induced colitic mice. Journal of Functional Foods 11, 438-448.

Josic, D., Persuric, Z., Resetar, D., Martinovic, T., Saftic, L. and Kraljevic Pavelic, S. (2017). Use of Foodomics for Control of Food Processing and Assessing of Food Safety. Adv Food Nutr Res 81, 187-229.

Karpas, Z. (2013). Applications of ion mobility spectrometry (IMS) in the field of foodomics. Food Research International 54, 1146-1151.

Kim, J. K., Ha, S. H., Park, S. Y., Lee, S. M., Kim, H. J., Lim, S. H., Suh, S. C., Kim, D. H. and Cho, H. S. (2012). Determination of lipophilic compounds in genetically modified rice using gas chromatography-time-of-flight mass spectrometry. Journal of Food Composition and Analysis 25, 31-38.

Korte, R. and Brockmeyer, J. (2017). Novel mass spectrometry approaches in food proteomics. TrAC Trends in Analytical Chemistry.

Kropf, U., Korošec, M., Bertoncelj, J., Ogrinc, N., Nečemer, M., Kump, P. and Golob, T. (2010). Determination of the geographical origin of Slovenian black locust, lime and chestnut honey. Food Chemistry 121, 839-846.

Lametsch, R., Roepstorff, P. and Bendixen, E. (2002). Identification of protein degradation during post-mortem storage of pig meat. Journal of Agricultural and Food Chemistry 50, 55085512.

Latorre, C. H., García, J. B., Martín, S. G. and Crecente, R. M. P. (2013). Chemometric classification of potatoes with protected designation of origin according to their producing area and variety. Journal of Agricultural and Food Chemistry 61, 8444-8451.

López-Rituerto, E., Savorani, F., Avenoza, A., Busto, J. H., Peregrina, J. M. and Engelsen, S. B. (2012). Investigations of la Rioja terroir for wine production using $1 \mathrm{H} N M R$ metabolomics. Journal of Agricultural and Food Chemistry 60, 3452-3461.

Manganaris, G. A., Goulas, V., Mellidou, I. and Drogoudi, P. (2017). Antioxidant Phytochemicals in Fresh Produce: Exploitation of Genotype Variation and Advancements in Analytical Protocols. Frontiers in Chemistry 5, 95. 
Marseglia, A., Acquotti, D., Consonni, R., Cagliani, L. R., Palla, G. and Caligiani, A. (2016). HR MAS1H NMR and chemometrics as useful tool to assess the geographical origin of cocoa beans - Comparison with HR1H NMR. Food Research International 85, 273-281.

Martinovic, T., Andjelkovic, U., Gajdosik, M. S., Resetar, D. and Josic, D. (2016). Foodborne pathogens and their toxins. Journal of Proteomics 147, 226-235.

Mazzei, P. and Piccolo, A. (2012). 1 H HRMAS-NMR metabolomic to assess quality and traceability of mozzarella cheese from Campania buffalo milk. Food Chemistry 132, 1620-1627.

Meplan, C., Johnson, I. T., Polley, A. C., Cockell, S., Bradburn, D. M., Commane, D. M., Arasaradnam, R. P., Mulholland, F., Zupanic, A., Mathers, J. C. and Hesketh, J. (2016). Transcriptomics and proteomics show that selenium affects inflammation, cytoskeleton, and cancer pathways in human rectal biopsies. Faseb j 30, 2812-25.

Montero, L., Herrero, M., Castro-Puyana, M. and Raquel, P. (2017). Trends in Analytical Chemistry Reprint of : Application of mass spectrometry-based metabolomics approaches for food safety, quality and traceability *. TrAC Trends in Analytical Chemistry 96, 62-78.

Morozova, O. and Marra, M. A. (2008). Applications of next-generation sequencing technologies in functional genomics. Genomics 92, 255-64.

Oms-Oliu, G., Odriozola-Serrano, I. and Martín-Belloso, O. (2013). Metabolomics for assessing safety and quality of plant-derived food. Food Research International. Elsevier.

Picariello, G., Mamone, G., Addeo, F., Nitride, C. and Ferranti, P. (2013). Proteomic-based Techniques for the Characterization of Food Allergens. In: Cifuentes, A. (ed.) Foodomics: Advanced Mass Spectrometry in Modern Food Science and Nutrition. pp 69-99. New Jersey: John Wiley \& Sons.

Pongsuwan, W., Fukusaki, E., Bamba, T., Yonetani, T., Yamahara, T. and Kobayashi, A. (2007). Prediction of Japanese green tea ranking by gas chromatography/mass spectrometrybased hydrophilic metabolite fingerprinting. Journal of Agricultural and Food Chemistry 55, 231-236.

Ren, G., Wang, S., Ning, J., Xu, R., Wang, Y., Xing, Z., Wan, X. and Zhang, Z. (2013). Quantitative analysis and geographical traceability of black tea using Fourier transform near-infrared spectroscopy (FT-NIRS). Food Research International 53, 822-826.

Resetar, D., Pavelic, S. K. and Josic, D. (2015). Foodomics for investigations of food toxins. Current Opinion in Food Science 4, 86-91.

Ríos-Reina, R., García-González, D. L., Callejón, R. M. and Amigo, J. M. (2018). NIR spectroscopy and chemometrics for the typification of Spanish wine vinegars with a protected designation of origin. Food Control 89, 108-116.

Ritota, M., Casciani, L., Han, B.-Z., Cozzolino, S., Leita, L., Sequi, P. and Valentini, M. (2012). Traceability of Italian garlic (Allium sativum L.) by means of HRMAS-NMR spectroscopy and multivariate data analysis. Food chemistry 135, 684-693.

Robert, C., Gillard, N., Brasseur, P. Y., Pierret, G., Ralet, N., Dubois, M. and Delahaut, P. (2013). Rapid multi-residue and multi-class qualitative screening for veterinary drugs in foods of animal origin by UHPLC-MS/MS. Food Additives and Contaminants Part a-Chemistry Analysis Control Exposure \& Risk Assessment 30, 443-457.

Rudkowska, I., Ponton, A., Jacques, H., Lavigne, C., Holub, B. J., Marette, A. and Vohl, M. C. (2011). Effects of a supplementation of $n-3$ polyunsaturated fatty acids with or without fish gelatin on gene expression in peripheral blood mononuclear cells in obese, insulinresistant subjects. J Nutrigenet Nutrigenomics 4, 192-202.

Ryan, J. M. (2017). Guide to food safety and quality during transportation : controls, standards and practices. Guide to Food Safety and Quality During Transportation, 1-313.

Rychlik, M., Kanawati, B. and Schmitt-Kopplin, P. (2017). Foodomics as a promising tool to investigate the mycobolome. Trac-Trends in Analytical Chemistry 96, 22-30.

Shabeer, T. P. A., Girame, R., Utture, S., Oulkar, D., Banerjee, K., Ajay, D., Arimboor, R. and Menon, K. R. K. (2018). Optimization of multi-residue method for targeted screening and 
quantitation of 243 pesticide residues in cardamom (Elettaria cardamomum) by gas chromatography tandem mass spectrometry (GC-MS/MS) analysis. Chemosphere 193, 447-453.

Shulaev, V. (2006). Metabolomics technology and bioinformatics. Brief Bioinform 7, 128-39.

Suman, S. P., Faustman, C., Stamer, S. L. and Liebler, D. C. (2007). Proteomics of lipid oxidationinduced oxidation of porcine and bovine oxymyoglobins. Proteomics 7, 628-640.

Tompkin, R. B. (2002). Control of Listeria monocytogenes in the food-processing environment. Journal of Food Protection 65, 709-725.

Trimigno, A., Marincola, F. C., Dellarosa, N., Picone, G. and Laghi, L. (2015). Definition of food quality by NMR-based foodomics. Current Opinion in Food Science 4, 99-104.

Turpin, W., Weiman, M., Guyot, J. P., Lajus, A., Cruveiller, S. and Humblot, C. (2018). The genomic and transcriptomic basis of the potential of Lactobacillus plantarum $A 6$ to improve the nutritional quality of a cereal based fermented food. International Journal of Food Microbiology 266, 346-354.

Valdés, A., Artemenko, K. A., Bergquist, J., García-Cañas, V. and Cifuentes, A. (2016). Comprehensive Proteomic Study of the Antiproliferative Activity of a PolyphenolEnriched Rosemary Extract on Colon Cancer Cells Using Nanoliquid ChromatographyOrbitrap MS/MS. Journal of Proteome Research 15, 1971-1985.

Valdés, A., Cifuentes, A. and León, C. (2017). Foodomics evaluation of bioactive compounds in foods. TrAC Trends in Analytical Chemistry 96, 2-13.

Valdes, A., Garcia-Canas, V., Artemenko, K. A., Simo, C., Bergquist, J. and Cifuentes, A. (2017). Nano-liquid Chromatography-orbitrap MS-based Quantitative Proteomics Reveals Differences Between the Mechanisms of Action of Carnosic Acid and Carnosol in Colon Cancer Cells. Mol Cell Proteomics 16, 8-22.

Valdes, A., Garcia-Canas, V., Simo, C., Ibanez, C., Micol, V., Ferragut, J. A. and Cifuentes, A. (2014). Comprehensive foodomics study on the mechanisms operating at various molecular levels in cancer cells in response to individual rosemary polyphenols. Anal Chem 86, 9807-15.

Valdes, A., Simo, C., Ibanez, C., Rocamora-Reverte, L., Ferragut, J. A., Garcia-Canas, V. and Cifuentes, A. (2012). Effect of dietary polyphenols on K562 leukemia cells: a Foodomics approach. Electrophoresis 33, 2314-27.

Valdés, A., Sullini, G., Ibáñez, E., Cifuentes, A. and García-Cañas, V. (2015). Rosemary polyphenols induce unfolded protein response and changes in cholesterol metabolism in colon cancer cells. Journal of Functional Foods 15, 429-439.

Vallverdu-Queralt, A. and Lamuela-Raventos, R. M. (2016). Foodomics: A new tool to differentiate between organic and conventional foods. Electrophoresis 37, 1784-94.

World Health Organization. (2015). Food safety [Online]. Available: http://www.who.int/mediacentre/factsheets/fs399/en/ [Accessed 06 April 2018].

Xu, Y.-J. and Wu, X. (2015). Foodomics in microbiological investigations. Current Opinion in Food Science 4, 51-55.

Yao, S., Li, T., Liu, H., Li, J. and Wang, Y. (2018). Traceability of Boletaceae mushrooms using data fusion of UV-visible and FTIR combined with chemometrics methods. Journal of the Science of Food and Agriculture 98, 2215-2222.

Yongtao, H., Le, S., Nan, Z., Ronghua, C., Yuhong, Q. and Canping, P. (2016). Multi-residue determination of 171 pesticides in cowpea using modified QuEChERS method with multi-walled carbon nanotubes as reversed-dispersive solid-phase extraction materials. Journal of Chromatography B 1031, 99-108.

Zhang, H., Wang, J., Li, L. and Wang, Y. (2017). Determination of 103 Pesticides and Their Main Metabolites in Animal Origin Food by QuEChERS and Liquid Chromatography-Tandem Mass Spectrometry. Food Analytical Methods 10, 1826-1843. 



\section{Figure captions:}

Figure 1. Schematic representation of the omic tools and areas of research covered by Foodomics. Redrawn from the cover of Trends in Analytical Chemistry (Foodomics and Modern Food Analysis issue, volume 96, November 2017) with permission from Elsevier.

Figure 2. Proteomics pipeline used for the identification, characterization and detection of species-specific peptide biomarkers for fish authentication purposes. Redrawn from (Carrera, Cañas and Gallardo, 2013) with permission from Elsevier.

Figure 3. A circular map of Salmonella Enteritidis transcripts detected by RNA-seq (with permission). The innermost circle shows nucleotide coordinates on the S. Enteritidis str. P125109 genome. From inside out: the two blue circles show genes expressed in $12 \mathrm{~h}$ and 312 h LB broth, respectively; the three red circles show genes expressed in $72 \mathrm{~h}, 216 \mathrm{~h}$, and $528 \mathrm{~h}$ peanut oil, respectively. Only genes detected in both biological replicates were shown. Desiccation-induced stress-response genes and non-coding RNAs were labeled at corresponding genomic locations. Genes, from A through L: dnaK, dnaJ, cspE, dps, cspD, sigE, grpE, sigma-32, major cold shock protein, ibpA, ibpB, and putative stress-response protein. Non-coding RNAs, from 1 to 9: sraC/ryeA RNA, rprA RNA, ryfA RNA, tke1 RNA, csrB RNA, RNase P RNA, sraJ RNA and csrC RNA. Redrawn from (Deng, Li and Zhang, 2012) with permission from Elsevier.

Figure 4. (A) Global Foodomics strategy used to investigate the activity of rosemary polyphenols against colon cancer HT29 cells at molecular level. (B) Foodomics identification of the proteins, genes and metabolites involved in three of the principal biological processes altered in HT29 colon cancer cells after the treatment with rosemary polyphenols. Underlined: down-regulated; no underlined: up-regulated. Redrawn from (Ibanez, Valdes, Garcia-Canas et al., 2012) with permission from Elsevier. 
Table 1. Some representative review papers on Foodomics covering applications in the three main areas discussed in this chapter, namely, i) food quality and traceability, ii) food safety and ii) food bioactivity $\&$ health.

Subject

Publication

year

Reference

Food Quality and Traceability

Antioxidant phytochemicals in fresh produce

Foodomics for quality control of food processing

Foodomics to differentiate organic and conventional

foods

Analytical chemistry methods in Foodomics

Definition of food quality by NMR-based Foodomics

\section{Food Safety}

Foodomics and food safety

Foodomics to investigate the mycobolome

Foodomics of foodborne pathogens and their toxins

Foodomics for investigations of food toxins

Foodomics: exploring safety, quality and bioactivity of foods

\section{Food Bioactivity \& Health}

Foodomics for human health

Food science, bioengineering, and medical innovation Foodomics evaluation of bioactive compounds in foods

Green Foodomics and bioactive compounds

Foodomics imaging by MS and NMR
2018 (Manganaris, Goulas, Mellidou and Drogoudi, 2017)

2017 (Josic, Persuric, Resetar et al., 2017)

2016 (Vallverdu-Queralt and LamuelaRaventos, 2016) (Gallo and Ferranti, 2016) (Trimigno, Marincola, Dellarosa, Picone and Laghi, 2015)

(Andjelković, Šrajer Gajdošik, GašoSokač, Martinović and Josić, 2017)

(Rychlik, Kanawati and SchmittKopplin, 2017)

2016 (Martinovic, Andjelkovic, Gajdosik, Resetar and Josic, 2016)

2015 (Resetar, Pavelic and Josic, 2015) (Cifuentes, 2015)

(Braconi, Bernardini, Millucci and Santucci, 2018)

(Bayram and Gokirmakli, 2018)

(Valdés, Cifuentes and León, 2017)

(Gilbert-López, Mendiola and Ibáñez, 2017)

(Canela, Rodriguez, Baiges, Nadal and Arola, 2016)

$2015 \quad$ (Xu and Wu, 2015)

